\title{
Singularly perturbed Burger-Huxley equation: Analytical solution through iteration
}

\author{
Deepmala Kamboj $^{1}$, M.D. Sharma ${ }^{2 *}$ \\ ${ }^{1}$ Mukand Lal National College, Yamuna Nagar, Haryana, INDIA \\ ${ }^{2}$ Department of Mathematics, Kurukshetra University, INDIA \\ *Corresponding Author e-mail: mdsharma@kuk.ac.in ; Tel +919896214489
}

\begin{abstract}
The work presented considers the initial boundary value problem for nonlinear singularly perturbed time dependent BurgerHuxley equation. The equation contains two terms with nonlinearities, the cubic term and the advection term. Generally, the severe difficulties of two types encounter in solving this problem. The first one comes from these nonlinearity terms and the second is due to the perturbation parameter in the diffusion term. When the perturbation parameter approaches zero, the problem exhibit boundary layers and most of conventional methods fails to capture this effect. A relatively different analytical technique called variational iteration method (VIM), is used to solve this equation. This method is able to obtain rapidly convergent successive approximations of exact solution without any restrictive approximations or the transformations that may change the physical behaviour of the problem. Numerical examples are studied to demonstrate the significance and effect of various parameters involved in the equation.
\end{abstract}

Keywords: Burger-Huxley equation, iteration method, analytical solution, nonlinearity, singularly perturbed, advection.

\section{Introduction}

Nonlinear phenomena occur in a wide variety of scientific applications such as plasma physics, solid state physics, fluid dynamics, chemical kinetics and mathematical biology (Albowitz and Clarkson, 1990). There are many nonlinear partial differential equations (PDEs), which are quite useful and applicable in engineering and physics such as well-known KdV equation (Kaya, 2003), MKdV equation, BBM equation, Burgers equation, KdV-KSV equation, RLW equation (Al-Khaled, 2005), and so on. Nonlinear wave phenomena of dispersion, dissipation, diffusion, reaction and convection are also represented through the nonlinear PDEs.

There exists a special class of nonlinear PDEs in which the coefficient of the highest order derivative term is very small. A member of this class is termed as a singularly perturbed nonlinear PDE and the small coefficient specifying the problem is identified as singular perturbation parameter. When this perturbation parameter (say, $\varepsilon$, hereafter) tends to zero, a breakdown occurs that results in the formation of layers in which the solution has a steep gradient. These layers are formed in the neighbourhood of the boundaries of the domain and are known as boundary layers. These PDEs represent the problems that involve a rapid change in the value of a physical variable over a limited region of space or time. Such problems, known as boundary-layer problems, are very common in physics and arise frequently in the studies of heat transfer with large Peclet numbers, Navier-Stokes flows with large Reynolds numbers, chemical reactor theory, simulation of oil extraction from underground reservoirs, aerodynamics, structural mechanics posed over thin dynamics, reaction-diffusion processes, quantum mechanics, optimal control, fluids dynamics, semiconductor device simulation, etc. Many of these problems are also used to model the situations in biosciences. For more details, readers are referred to Roos et al. (1996). In this reference, the conditions are also discussed for the existence and uniqueness of solutions of such problems. 
For the higher values of $\varepsilon$, number of solution methodologies exist in the literature (Cole, 1951; , Evans and Abdullah, 1984; Hopf, 1950; Ozis et al., 2003). But, for sufficiently small $\varepsilon$, the existing solution methodologies fail and a discrepancy occurs (Zang et al., 1997). Numerical analysis and asymptotic analysis are the two principal approaches that are used mainly to obtain the solutions of singularly perturbed nonlinear differential equations. The method of matched asymptotic expansion and the method of multiple scales are the techniques used mainly in asymptotic analysis (Eckhaus, 1973, 1979; Nayfeh, 1973). But, there can be some difficulties in applying these asymptotic expansion methods. For example, finding the appropriate expansion in the inner and the outer regions are not a routine exercise, but it requires skill, insight and experimentation. Then, one is left only with numerical methods. Several numerical methods have been developed for singularly perturbed nonlinear differential equations. These methods include finite difference methods, spline approximation methods, finite element methods, shooting methods, collocation methods, etc (Kadalbajoo and Patidar, 2002; Ringhofer, 1984; Stynes and O'Riordan, 1986). Khattak (2009) used a computational mesh-less method, based on collocation method using radial basis functions, to solve the generalized Burger-Huxley equation. Unfortunately, all these numerical techniques have their in-built deficiencies. For example, standard numerical methods with uniform mesh gives unsatisfactory results as the boundary layer narrows with smaller $\varepsilon$. It requires a refined mesh to accumulate the solution within the boundary layer, which increases the computational work a lot. The numerical techniques involving discretization of variables also give rise to rounding off errors. Reducing nonlinear equations to linear requires quasi-linearization process, which is not easy to implement. Moreover, it provides the solution at the grid points only and requires an interpolation to find the solution at other points. An alternative can be a procedure to calculate the approximate solution of analytical or differential equations through iterative process, for example, variational iteration method (referred, hereafter, as VIM) proposed by He (1999). It is recognized that this method can be an effective procedure for solution of various deterministic problems in physics, biology and chemical reactions. The efficiency of the method has been proved, formally, in many recent studies (He, 1998a, 1998b, 2006, 2007; Tatari and Dehghan, 2007; El-Wakil and Abdou, 2008; Shakeri and Dehghan, 2008). Batiha et al. (2007, 2008) used VIM for solving generalized Burgers-Huxley equation but this equation is not singularly perturbed i.e. perturbation parameter is not taken in consideration.

Inspired and motivated by the simple and effective application of this method, the present study aims to explore the applicability of VIM in solving the general form of a time dependent Burger-Huxley equation, analytically. This equation represents a prototype model to describe the interactions between reaction mechanisms, convection effects, and diffusion transports, which has been investigated extensively in Satsuma (1987). The basic concept of the variational iteration method is illustrated in section 2. The detailed formulation of the problem considered and the derivation of solution through VIM is explained in section 3 . In the section 4, numerical examples are solved for various physically significant models, which are deduced from the general form of BurgerHuxley equation. The numerical calculation aims to analyse the effects of various parameters on the approximate solution of the PDE. This approximate solution is computed for different values of perturbation parameter $(\varepsilon)$. It is to observe the layer behavior of the solution for smaller values of $\varepsilon$ leading to singular perturbations. The numerical calculation aims to analyse the effects of various parameters on the approximate solution of the PDE. This approximate solution is computed for different values of perturbation parameter $(\varepsilon)$. It is to observe the layer behavior of the solution for smaller values of $\varepsilon$ leading to singular perturbations. The section 5 contains the remarks on the numerical results discussed in the section 4.

\section{Variational iteration method}

To illustrate the basic concept of the variational iteration method, the following general nonlinear system is considered

$$
L[u(x)]+N[u(x)]=g(x),
$$

where $L$ is a linear operator, $N$ is a nonlinear operator and $g(x)$ is a given continuous function. According to the variational iteration method, a correctional functional is constructed in the form

$$
u_{n+1}(x)=u_{n}(x)+\int_{0}^{x} \lambda(s)\left[L u_{n}(s)+N \tilde{u}_{n}(s)-g(s)\right] d s,
$$

where, $u_{n}(n=0,1,2, \ldots$.$) , denotes the solution after the respective (say, \mathrm{n}$ ) number of iterations. ' $\sim$, over a quantity restricts its variation, i.e. $\delta \tilde{u}_{n}=\delta \tilde{u}_{n x}=\delta \tilde{u}_{n x x}=0$. The function $\lambda(s)$ is a general Lagrange multiplier (Inokuti et al., 1978), which can be identified optimally via variational theory. The successive approximations $u_{n+1}(x)$ of the solution $u$, for positive integer n, are readily obtained by using the determined Lagrangian multiplier and any selective function $u_{0}$. Consequently, the exact solution may be obtained by using

$$
u(x)=\lim _{n \rightarrow \infty} u_{n}(x) .
$$

For the convergence of this sequence and its rate, the Banach's theorem is recalled:

Banach's Fixed Point Theorem: Assume that $X$ is a Banach space, 
is a nonlinear mapping, and suppose that

$$
A: X \rightarrow X
$$

$$
\|A[u]-A[\bar{u}]\| \leq \gamma\|u-\bar{u}\|, \forall u, \bar{u} \in X
$$

for some constant $\gamma<1$. Then $A$ has a unique fixed point. Furthermore, the sequence

$$
u_{n+1}=A\left[u_{n}\right]
$$

with an arbitrary choice of $u_{0} \in X$ converges to the fixed point of $A$ and

$$
\left\|u_{k}-u_{l}\right\| \leq\left\|u_{1}-u_{0}\right\| \sum_{j=l}^{k-1} \gamma^{j}
$$

According to the above theorem, for the nonlinear mapping

$$
A[u]=u_{n}(x)+\int_{0}^{x} \lambda(s)\left[L u_{n}(s)+N \tilde{u}_{n}(s)-g(s)\right] d s,
$$

a sufficient condition for the convergence of the variational iteration method is the strict contraction of $A$ (Tatari and Dehghan, 2007). Furthermore, sequence $u_{n+1}=A\left[u_{n}\right]$ converges to the fixed point of $A$, which is also the solution of the nonlinear system considered above. In the above theorem, the rate of convergence depends on $\gamma$ and therefore, in the variational iteration method, the rate of convergence depends on $\lambda$.

\section{Formulation of the Problem and Solution}

From mathematical prospective, a general form of Burger-Huxley equation is a singularly perturbed nonlinear PDE, given by

$$
u_{t}+\alpha u u_{x}-\varepsilon u_{x x}-\beta(1-u)(u-\gamma) u=0, x \in(0,1) \text {, }
$$

where subscripts ' $x$ ' and ' $t$ ' denotes partial derivatives with respect to space and time respectively. The parameters $\alpha, \beta \geq 0$, $\gamma \in(0,1)$ and $0<\varepsilon<<1$, define the aspects of interactions in various fields in the problem. In the present study, the equation (1) is solved subject to the boundary conditions, given by

$$
u(x, 0)=\sin (\pi x), \quad u(0, t)=u(1, t)=0,0<t \leq T .
$$

The problem is considered to be boundary value problem where as in Batiha et al. (2007, 2008) initial value problem is taken with different initial conditions.

Following the procedure of VIM in section 2, a correction functional is constructed as follows.

$$
u_{n+1}(x, t)=u_{n}(x, t)+\int_{0}^{t} \lambda(s, t)\left[u_{n s}+\alpha \tilde{u}_{n} \tilde{u}_{n x}-\varepsilon \tilde{u}_{n x x}-\beta\left(1-\tilde{u}_{n}\right)\left(\tilde{u}_{n}-\gamma\right) \tilde{u}_{n}\right] d s,
$$

The value of Lagrangian multiplier $\lambda(s, t)$, in this case, is chosen to make correction functional (3) stationary, i.e., we must have

$$
\delta u_{n+1}=\delta u_{n}+\delta \int_{0}^{t} \lambda(s, t)\left[u_{n s}+\alpha \tilde{u}_{n} \tilde{u}_{n x}-\varepsilon \tilde{u}_{n x x}-\beta\left(1-\tilde{u}_{n}\right)\left(\tilde{u}_{n}-\gamma\right) \tilde{u}_{n}\right] d s=0
$$

Solving the integral once yields

$$
\delta u_{n+1}=\delta u_{n}\left(1+\left.\lambda(s, t)\right|_{s=t}\right)-\int_{0}^{t} \frac{\partial \lambda}{\partial s} \delta u_{n} d s=0,
$$

giving the conditions for stationary correctional functional (3) as follows.

$$
\frac{\partial \lambda}{\partial s}=0, \quad 1+\left.\lambda(s, t)\right|_{s=t}=0
$$

Solving these conditions, the Lagrange multiplier is obtained as

$$
\lambda(s, t)=-1 \text {. }
$$

Substituting this value of $\lambda$ into (3) gives a relation

$$
u_{n+1}(x, t)=u_{n}(x, t)-\int_{0}^{t}\left[u_{n s}+\alpha \tilde{u}_{n} \tilde{u}_{n x}-\varepsilon \tilde{u}_{n x x}-\beta\left(1-\tilde{u}_{n}\right)\left(\tilde{u}_{n}-\gamma\right) \tilde{u}_{n}\right] d s,
$$

which is an iteration formula to calculate the successive approximations to the solution of the problem (1) - (2) . This iteration formula can be rewritten in a computation-convenient format as

$$
u_{n+1}(x, t)=u_{n}(x, t)+I_{n, 1}+I_{n, 2}+I_{n, 3}+I_{n, 4}+I_{n, 5}+I_{n, 6},
$$


where

$$
\begin{aligned}
& I_{n, 1}=-\int_{0}^{t} u_{n s} d s, \\
& I_{n, 2}=-\alpha \int_{0}^{t} u_{n} u_{n x} d s, \\
& I_{n, 3}=\varepsilon \int_{0}^{t} u_{n x x} d s, \\
& I_{n, 4}=-\beta \gamma \int_{0}^{t} u_{n} d s, \\
& I_{n, 5}=\beta(1+\gamma) \int_{0}^{t} u_{n}^{2} d s, \\
& I_{n, 6}=-\beta \int_{0}^{t} u_{n}^{3} d s .
\end{aligned}
$$

Using these integrals, the first approximation to the solution of (1) - (2) can be written as

$$
u_{1}=u_{0}+I_{0,1}+I_{0,2}+I_{0,3}+I_{0,4}+I_{0,5}+I_{0,6} \text {, }
$$

where $u_{0}$ represents the initial approximation. Starting with $u_{0}=u(x, 0)=\sin (\pi x)$ and resulting expressions for $I_{0, k}$, $k=1,2,3, . ., 6$ in (5) are computed. Using these expressions in (6), the first approximation $u_{1}$ of the solution is expressed as

$$
u_{1}=f_{0}+f_{1} t
$$

Where

$$
\begin{gathered}
f_{0}=\sin (\pi x), \\
f_{1}=-\alpha f_{0} f_{0 x}+\varepsilon f_{0 x x}-\beta \gamma f_{0}+\beta(1+\gamma) f_{0}^{2}-\beta f_{0}^{3} .
\end{gathered}
$$

Consequently, the relation

$$
u_{2}=u_{1}+I_{1,1}+I_{1,2}+I_{1,3}+I_{1,4}+I_{1,5}+I_{1,6} \text {. }
$$

enables to derive the second approximation $u_{2}$ of the solution from $u_{1}$. With $u_{1}$ given by (7), the integrals $I_{1, k},(k=1,2,3, . ., 6)$ are evaluated. Using these evaluations in (10) yields the second approximation to the solution, given by

$$
u_{2}=f_{0}+f_{1} t+f_{2} t^{2}+f_{3} t^{3}+f_{4} t^{4}
$$

In this expression, $f_{0}, f_{1}$ are same as in $(8)-(9)$, and

$$
\begin{aligned}
& f_{2}=-\frac{\alpha}{2}\left(f_{0} f_{1 x}+f_{1} f_{0 x}\right)+\frac{\varepsilon}{2} f_{1 x x}-\frac{\beta \gamma}{2} f_{1}+\beta(1+\gamma) f_{0} f_{1}-\frac{3 \beta}{2} f_{0}^{2} f_{1}, \\
& f_{3}=-\frac{\alpha}{3} f_{1} f_{1 x}+\frac{\beta(1+\gamma)}{3} f_{1}^{2}-\beta f_{0} f_{1}^{2}, \\
& f_{4}=-\frac{\beta}{4} f_{1}^{3} .
\end{aligned}
$$

In (11), $f_{0}, f_{1}, f_{3}, f_{4}=0$ for any value of $\alpha, \beta, \gamma$ and $\varepsilon$ at $x=0$. But $f_{2}$ is not identically zero but depends on $\varepsilon$ and $\beta$. However, for fixed values of $\alpha, \gamma$ and also for small values of $t$, we get $u_{2} \approx 0$ for small values of $\varepsilon$ and $\beta$. Presence of the term $\frac{\varepsilon}{2} f_{1 x x}$ in $f_{2}$ is responsible for this break-up of boundary conditions. Presence of the term $\frac{\varepsilon}{2} f_{1 x x}$ in $f_{2}$ is responsible for this break-up of boundary conditions, no such term is present in solution given by Batiha et al. (2007, 2008). The next approximation to the solution is obtained with a similar use of the iteration formula (4) for the most recent approximation of the solution. The sequence of these successive approximations will be converging rapidly to the accurate solution. Simple expressions can not be expected for the higher approximation to the solution. Hence, the closed form of the approximate solution may not be analysed for a possible convergence to the actual root. Hence, in the next section, numerical examples are considered to explain the changes in the behaviour of successive approximate solutions. 


\section{Examples and Discussion of Numerical Results}

The introduction of the term ' $\varepsilon u_{x x}$ ' in Burger-Huxley equation represents the presence of small isotropic diffusion in the reaction mechanism. Then the problem represented by PDE with the highest order derivative term multiplied by a small parameter $\varepsilon$ should be exhibiting a multi-scale character. In case of large values of $\varepsilon$, the dominating diffusion takes over the nonlinearity effect in the problem. It results in the smoothening of the solution in short intervals of time as well as in narrow intervals of space. Further, the first order terms, which are expected to produce shocks, may not matter much. On the other hand, when $\varepsilon \rightarrow 0$, the diffusion term interacts with lower order terms and it results in a breakdown of singular perturbation. Then the solution of the perturbed problem no longer remains smooth in short interval of time but still exhibits a layer behavior. These are a kind of PDEs that encounter frequently in fluid dynamics and in the description of many non-linear wave phenomena. No such work is explained in Batiha et al. $(2007,2008)$.

In Burger-Huxley equation (1), the parameter $\alpha$ characterizes a non-linear transfer and the parameters $\beta$ and $\gamma$ are used to describe a nonlinear source. For computational work, values chosen for these parameters are given by $\alpha=1, \beta=1$ and $\gamma=0.5$. For these parameter values, the second approximation to the exact solution in (11) is given by

$$
\begin{aligned}
u \approx f_{0}+f_{1} t+\{ & \left.-.5\left(f_{0} f_{1 x}+f_{1} f_{0 x}\right)+.5 \varepsilon f_{1 x x}-.25 f_{1}+1.5 f_{0} f_{1}-1.5 f_{0}^{2} f_{1}\right\} t^{2} \\
& +\left(-\frac{1}{3} f_{1} f_{1 x}+.5 f_{1}^{2}-f_{0} f_{1}^{2}\right) t^{3}-.25 f_{1}^{3} t^{4},
\end{aligned}
$$

where

$$
f_{0}=\sin (\pi x)
$$

$$
f_{1}=-f_{0} f_{0 x}+\varepsilon f_{0 x x}-.5 f_{0}+1.5 f_{0}^{2}-f_{0}^{3} \text {. }
$$

This approximate solution is computed for different values of $\varepsilon$. It is observed that the solution exhibits a layer behavior for small values of $\varepsilon$, as shown in Figure 1 . This behaviour is very much in agreement with that observed by Kaushik and Sharma (2008). For the limiting $\varepsilon \rightarrow 0$, this particular case of PDE (1) becomes nonlinear hyperbolic and its solution is discussed later in this study for some particular models. It is observed that at any given time $(t)$, the solution is parabolic with $X$ with maximum around $x=0.5$. The solution is nearly symmetric with respect to the maximum. On the other hand for any given $x$, the solution variations with $t$ are nearly linear. Maximum of the solution $u(x, t)$ is observed at the largest $t$, i.e., $t=0.1$. The solution value increases a little with decrease of $\varepsilon$ from 0.1 to 0.01 . However, any further decrease in $\varepsilon$ may not have any effect on the solution.
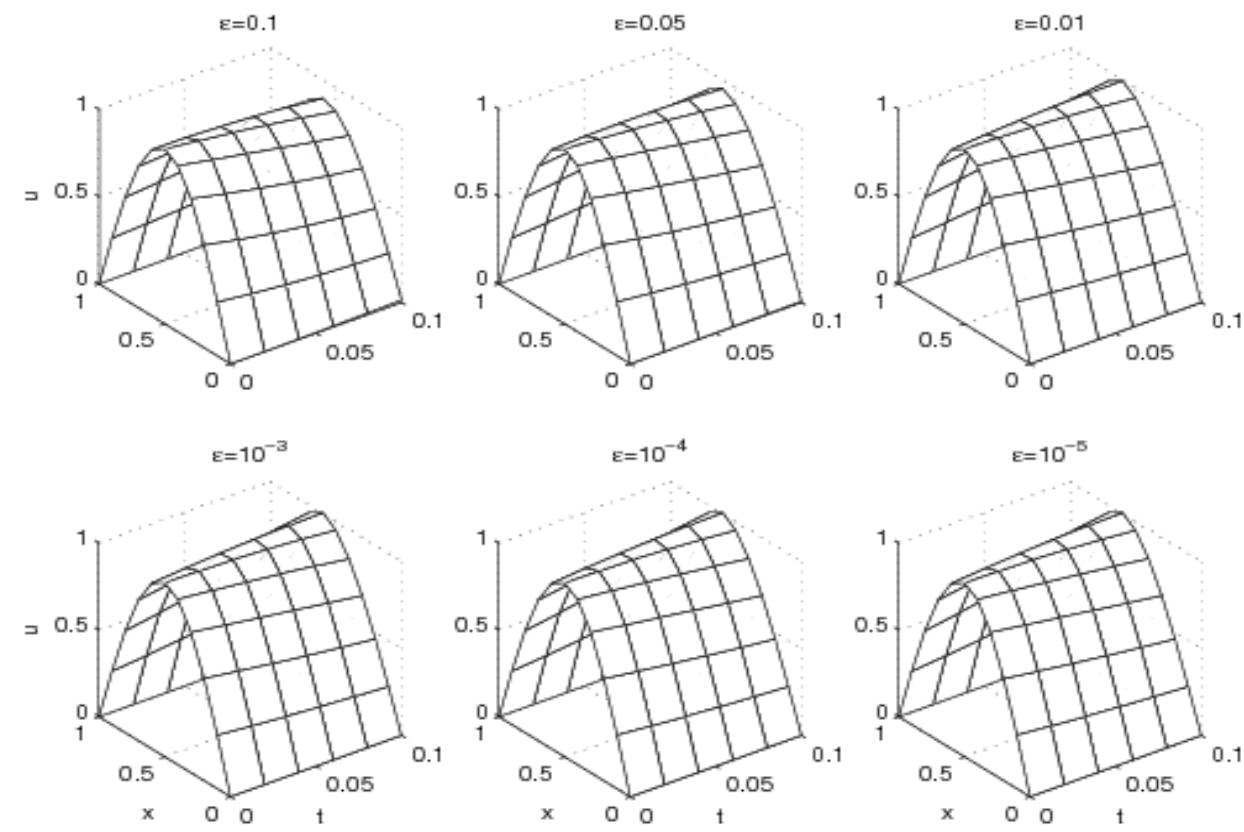

Figure 1. Solution $u(x, t)$ of singularly perturbed Burger-Huxley equation for values of parameter $\alpha=\beta=\gamma=1$ and for different values of $\varepsilon$ 
The solution $u(x, t)$ computed above and displayed in Figure 1 contains the contributions from different terms in the PDE (1). These contributions are represented by the parameters $\gamma, \beta$ and $\alpha$. The roles of these parameters in changing the solution are explained through the Figures 2 to 4 for two small values of $\varepsilon=0.1,0.001$.
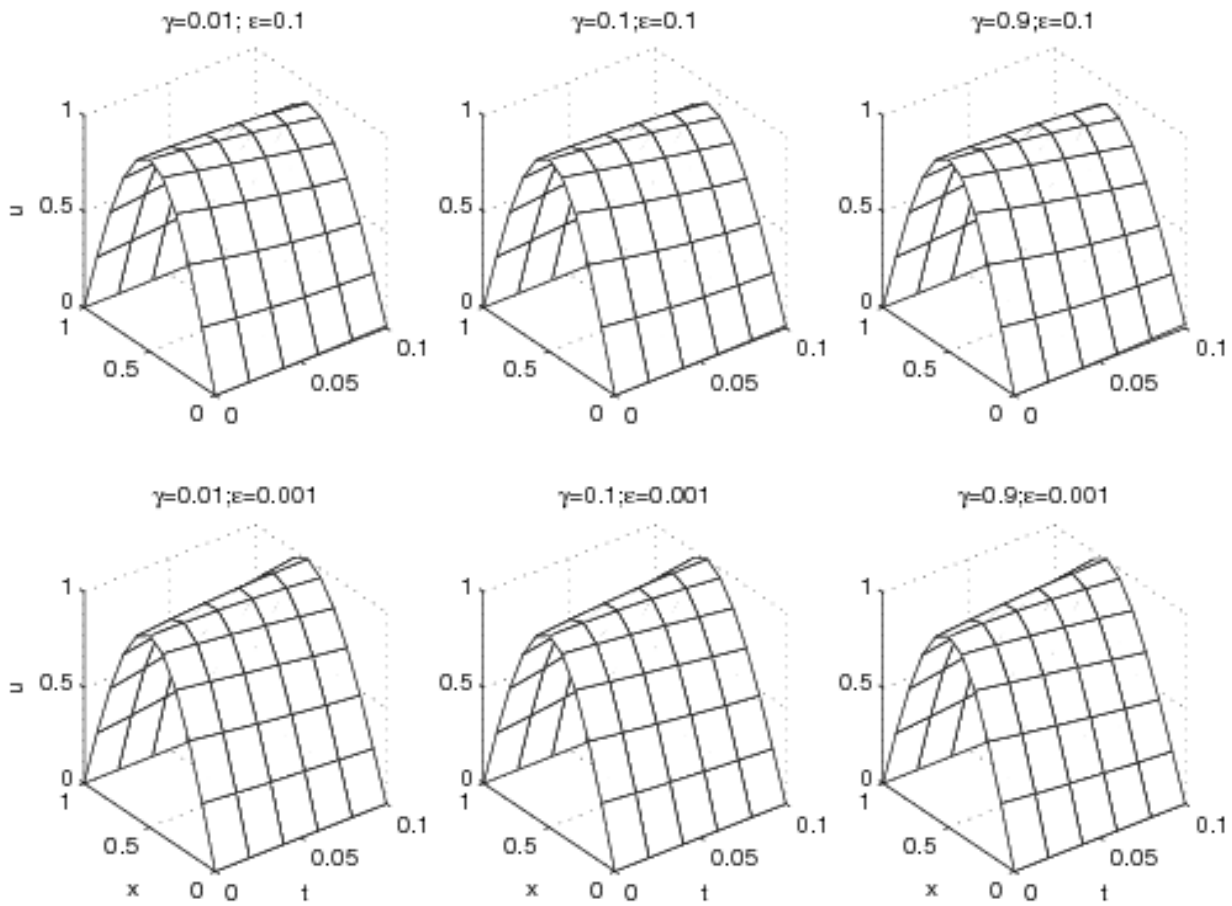

Figure 2. Solution $u(x, t)$ of singularly perturbed Burger-Huxley equation for $\alpha=\beta=1 ; \gamma=0.01,0.1,0.9$ and $\varepsilon=0.001,0.1$

Figure 2: The solution is computed for $\alpha=\beta=1$ and $\varepsilon=0.001,0.1$. Three value of $\gamma=0.01,0.1,0.9$; are chosen to observe the variations of solution with $\gamma$. It is observed that the change in $\gamma$ has almost no effect on the solution when $\varepsilon=0.1$ but a very little effect is observed for smaller $\varepsilon=0.001$.
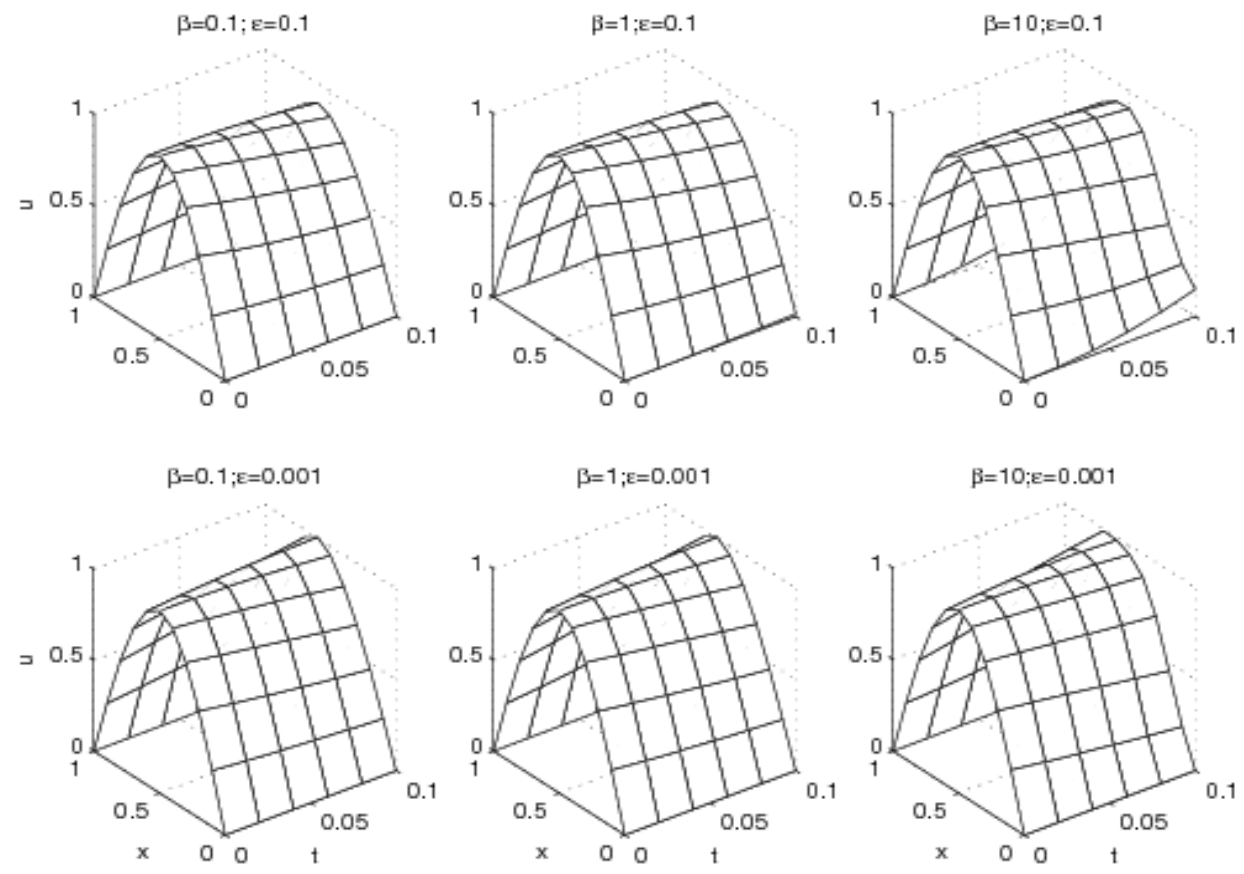

Figure 3. Solution $u(x, t)$ of singularly perturbed Burger-Huxley equation for $\alpha=1, \gamma=0.5 ; \beta=0.1,1,10$ and $\varepsilon=0.001,0.1$ 
Figure 3: The role $\beta$ in varying the solution $u(x, t)$ is explained through the plots in the Figure 3 for $\beta=0.1,1,10$ and $\varepsilon=0.001,0.1$. The values chosen for other parameters are $\gamma=0.5 \alpha=1$. The role of $\beta$ in changing the solution is quite similar to that of $\gamma$ observed in Figure 2. The only exception is that for larger values of $\beta$ along with $\varepsilon=0.1$, the solution may not be satisfying the boundary condition $u(0, t)=0$ for $t>0.2$.
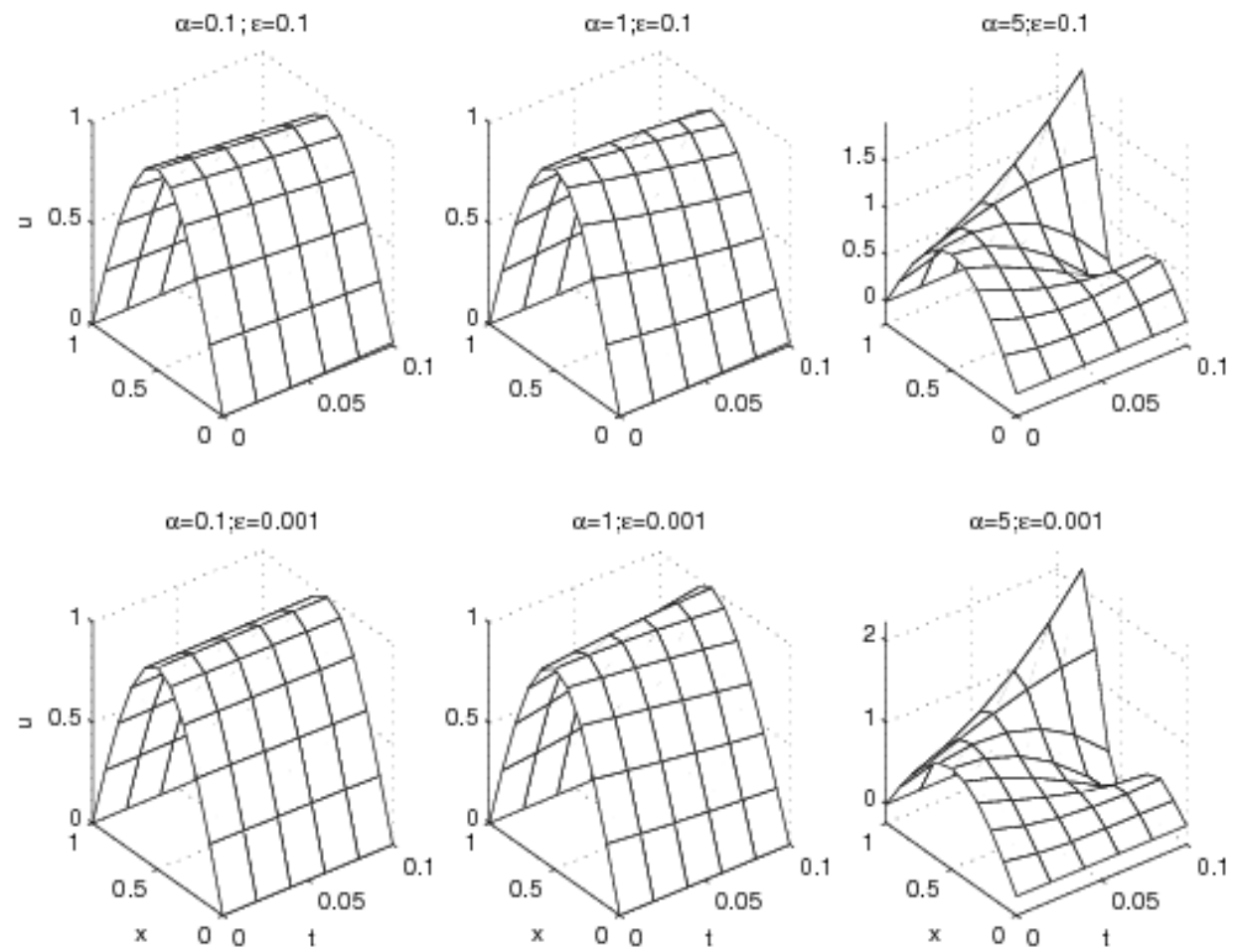

Figure 4. Solution $u(x, t)$ of singularly perturbed Burger-Huxley equation for $\beta=1, \gamma=0.5 ; \alpha=0.1,1,5$ and $\varepsilon=0.001,0.1$

Figure 4: In this figure the various plots exhibit the variations of solution $u(x, t)$ for $\alpha=0.1,1,5$ and $\varepsilon=0.001,0.1$. Values $\beta=1, \gamma=0.5$ define other parameters. A little change in solution is observed for variations of non-linearity parameter $\alpha \in(0.1,1)$. But it may not be considered much significant when compared to the change in solution for $\alpha$ increasing beyond 1. It is observed that for $\alpha>>1$, the layer behavior vanishes and the approximated solution may abandon the boundary condition $u(1, t)=0$ for $t>0.2$.

\subsection{Particular Cases}

There exists some particular sets of values for the parameters involved in the general PDE (1). For these sets, the equation (1) reduces to models having great physical significance. The particular cases for parameters involved are not discussed in Batiha et al. $(2007,2008)$. Solutions for four such models are discussed as follows.

Model 1: $\alpha=0, \varepsilon=\beta=1$ : For $\gamma \in(0,1)$, the reduced equation (1) becomes FitzHugh-Nagumo equation, i.e.,

$$
u_{t}-u_{x x}-(1-u)(u-\gamma) u=0 \text {, }
$$

where $X$ and $t$ denote rescaled variables for space and time, respectively. The variations of solution $u$ with $X$ are displayed in the Figure 5, for six values of non-dimensional time $t=0,0.02,0.04,0.06,0.08,0.1$. It is observed that the delayed solutions are reduced significantly. However, the rate of decay of solution with time decreases with the increase of $t$, i.e. deceleration with time. It is also noted that the delayed solutions may deviate a bit from the requirement of homogeneous boundary conditions at both the ends. 


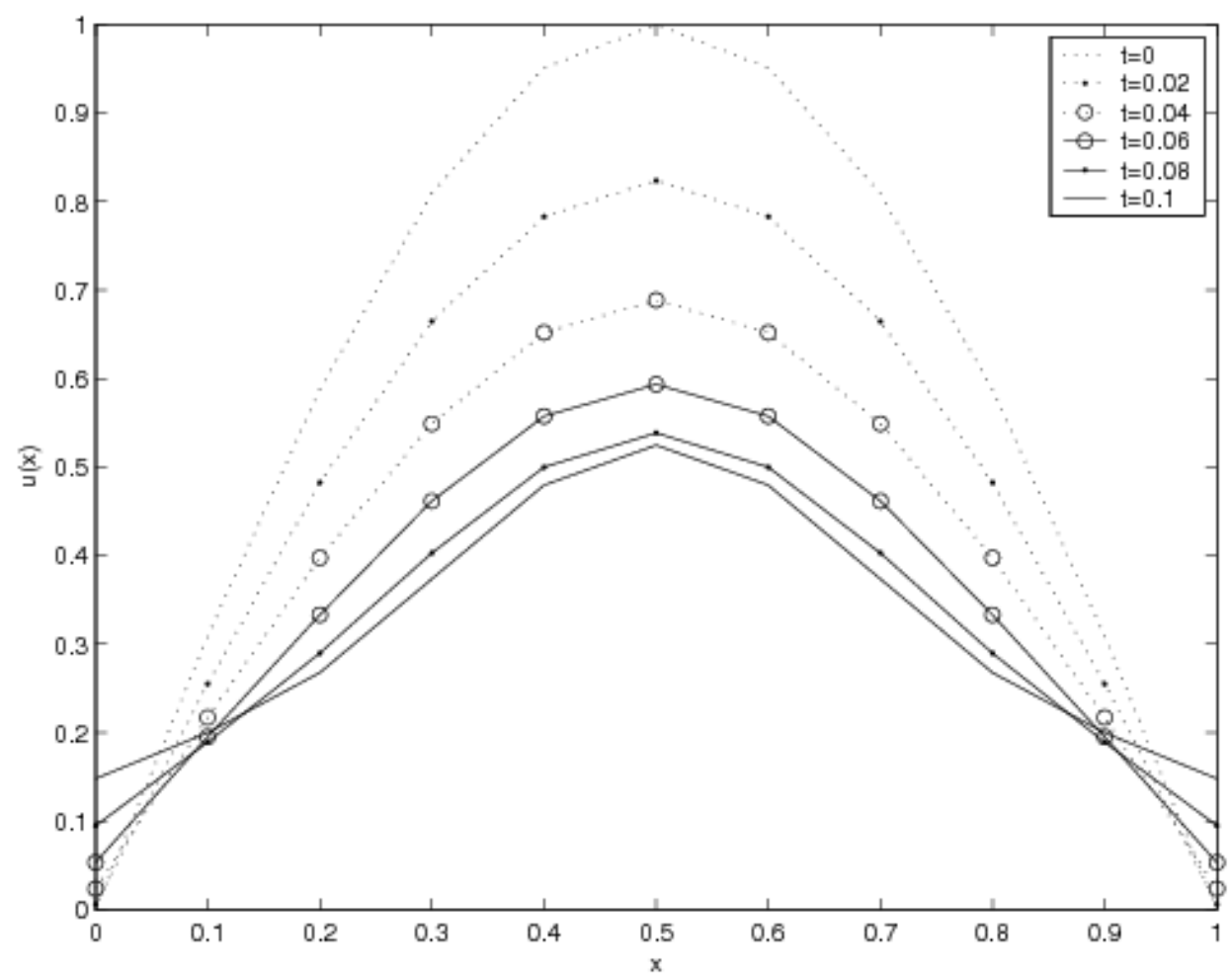

Figure 5. Solution of FitzHugh-Nagumo equation ( $\gamma=.5, \alpha=0$, and $\varepsilon=\beta=1$ )

This equation contains the key features of Hodgkin-Huxley model and is used to describe the propagation of electrical pulse in a neuron. The solution term $u$ denotes the transmembrane potential in the axon and the term $u_{x x}$ represents the activation part. In many cases, the peaks of the activating function correspond to the locations of action potential generation for a neuron in a uniform electric field. This allows one to predict the site of action potential initiation in a spatially complicated neuron without having to simulate the full Hodgkin-Huxley dynamics. The equation (13) is a simplification of the Hodgkin-Huxley equation that was derived as a model for the propagation of action potentials in the giant nerve axon of the squid. It was the first model to correctly describe the ionic basis of excitation. Many fascinating phenomena such as bursting oscillator (Duan and Lu, 2006), interspike (Liu et al., 2005), bifurcation and chaos (Zhang et al., 2006), are also associated with this equation.

Now, for $\alpha=0, \beta=\varepsilon=1$, the expression (11) reduces to define the solution of FitzHugh-Nagumo equation, given by

$$
u(x, t) \approx f_{0}+f_{1} t+\left(.5 f_{1 x x}-.5 \gamma f_{1}+(1+\gamma) f_{0} f_{1}-1.5 f_{0}^{2} f_{1}\right) t^{2}+\left(\frac{1}{3}(1+\gamma) f_{1}^{2}-f_{0} f_{1}^{2}\right) t^{3}-.25 f_{1}^{3} t^{4}
$$

where

$$
f_{1}=f_{0 x x}-\gamma f_{0}+(1+\gamma) f_{0}^{2}-f_{0}^{3}
$$

This analytical solution is computed for a particular value of $\gamma=0.5$ and is plotted in Figure 5. For $\gamma=0.001$ and initial approximation $u_{0}=\left(\frac{\gamma}{2}+\frac{\gamma}{2} \tanh \left(A_{1} x\right)\right)$, where $A_{1}=\frac{-\alpha+\sqrt{\alpha^{2}+8 \beta}}{8} \gamma$, the solution (14) finds an agreement with the exact solution (Wang et al., 1990; Ismail et al., 2004).

A qualitative analysis of the Hodgkin-Huxley model (Hodgkin and Huxley, 1952a, b), which closely mimics the ionic process at a real nerve membrane, is performed by means of singular perturbation theory. This was achieved by introducing a perturbation parameter. A decrease in parameter "speeds up" the fast variables (membrane potential and sodium activation) of Hodgkin-Huxley equation but it does not affect the slow variables (sodium inactivation and potassium activation). Similarly, equation (13) with the introduction of $\varepsilon$ represents the singularly perturbed equation of FitzHugh-Nagumo type (FitzHugh, 1961; Nagumo, 1962; Sleeman, 1982), i.e.,

$$
u_{t}-\varepsilon u_{x x}-(1-u)(u-\gamma) u=0
$$


In order to discuss the moments of fronts and backs, the problem is formally reduced by taking the limit $\varepsilon \rightarrow 0$. The presence of this singular limit requires two different scaling for analysis. The outer scaling is obtained through $\varepsilon=0$. It indicates that the transmembrane potential resides on one of the two solution branches. The inner scaling is obtained for $\varepsilon \in(0,1)$.

The solution branches define the traveling waves, which play the role of the boundary layers. This technique restricts the analysis to the perturbations that travel with the same velocity as the wave. Even if the traveling wave pulse has a triggered wave back, it is possible that after a perturbation, the back might transiently convert to a phase wave. It turns out, by allowing $\gamma$ to cross 0 and 1 . Hence, the singularly perturbed system for traveling waves possesses turning points. The conceptual procedure for constructing fronts, backs and pulse solutions of certain reaction-diffusion systems by a singular perturbation analysis was brought out by Feinn and Ortoleva (1977).

Model 2: $\alpha=1, \beta=0$ : The equation (1) reduces to Burger equation, at high Reynolds number $\operatorname{Re}\left(=\frac{1}{\varepsilon}\right)$ that establishes a balance between time evolution, nonlinearity and diffusion. The equation is given by

$$
u_{t}+u u_{x}-\varepsilon u_{x x}=0
$$

where $u(x, t)$ denotes flow velocity. The variations of flow velocity with $x \in[0,1]$ are exhibited in the Figure 6 , for some discrete values of $t \in[0,0.1]$ these solutions are computed for three values of singular parameter $\varepsilon=0.001,0.01,0.1$. However, the change of $\varepsilon \in(0.001,0.1)$ does not have any significant effect on the solution $u(x)$, at any given time $t$.

Burger equation (16) is a fundamental nonlinear PDE of second order to describe the integrated process of convection-diffusion in physics (Bateman, 1915; Burgers, 1948). It occurs in various areas of turbulence, boundary layer behaviour, shock wave formation and mass transport, etc. The convection-diffusion term, which represents the main factors to influence the fluid flow in Navier-Stokes equation are included in Burger equation while the pressure is neglected. One of the major source of difficulties in Navier-Stokes equation is the inviscid boundary layers produced by steepening effect of the nonlinear advection term. The same difficulty is also encountered in Burger equation. For this reason, the analytic solvability of the Burger model is an important problem of fluid dynamics. Hopf (1950) and Cole (1951) solved Burger equation analytically for arbitrary initial conditions. In many cases, these solutions involve infinite series which may converge very slowly for small values of $\varepsilon$, which corresponds to steep wave fronts in the propagation of the dynamics wave forms. Hopf (1950) and Cole (1951) discovered transformations that reduce the Burger equation to linear heat equation. However, an infinite domain $-\infty<x<\infty$ was required for these transformations. Hence, these transformations can not be used for many physical applications defined only in a finite interval.

For $\alpha=1, \beta=0$ the solution (11) reduces to

$$
u \approx f_{0}+f_{1} t+\left\{-.5\left(f_{0} f_{1 x}+f_{1} f_{0 x}\right)+.5 \varepsilon f_{1 x x}\right\} t^{2}-\left(\frac{1}{3} f_{1} f_{1 x}\right) t^{3}
$$

where

$$
f_{1}=-f_{0} f_{0 x}+\varepsilon f_{0 x x}
$$

This is the analytical solution for Burger equation, which is in agreement with (Cole, 1951). The variations of the solution $u(x, t)$ for different values of $\varepsilon$ are shown in Figure 6.

Model 3: $\alpha=1, \beta=0, \varepsilon \rightarrow 0$ : The equation (1) reduces to momentum equation of gas, describing the velocity $u$, transported by the fluid motion itself,

$$
u_{t}+u u_{x}=0
$$

First systematically study of this equation was performed by Poisson and Riemann in the early nineteenth century. Since it matters in so many applications, this equation appears in the literature under a variety of names, viz., including the Riemann equation, the inviscid Burger equation and the dispersion-less Korteweg-de-Vries equation. The equation itself and its multidimensional and multi-component generalizations play crucial roles in modeling of gas dynamics, traffic flow, flood waves in rivers, chromatography, chemical reactions and many other areas.

Using $\alpha=1, \beta=0$ and $\varepsilon \rightarrow 0$, the solution (11) reduces to

$$
u \approx f_{0}+f_{1} t-.5\left(f_{0} f_{1 x}+f_{1} f_{0 x}\right) t^{2}-\left(\frac{1}{3} f_{1} f_{1 x}\right) t^{3},
$$

where

$$
f_{1}=-f_{0} f_{0 x}
$$


The solution (19) of Riemann equation is plotted in the Figure 7. It is observed that the decay of solution with time in first half of $X$-domain get reversed in the second half. This implies the existence of a small interval around the middle of domain of $x$ where the decay of solution with time may be absent or very small.
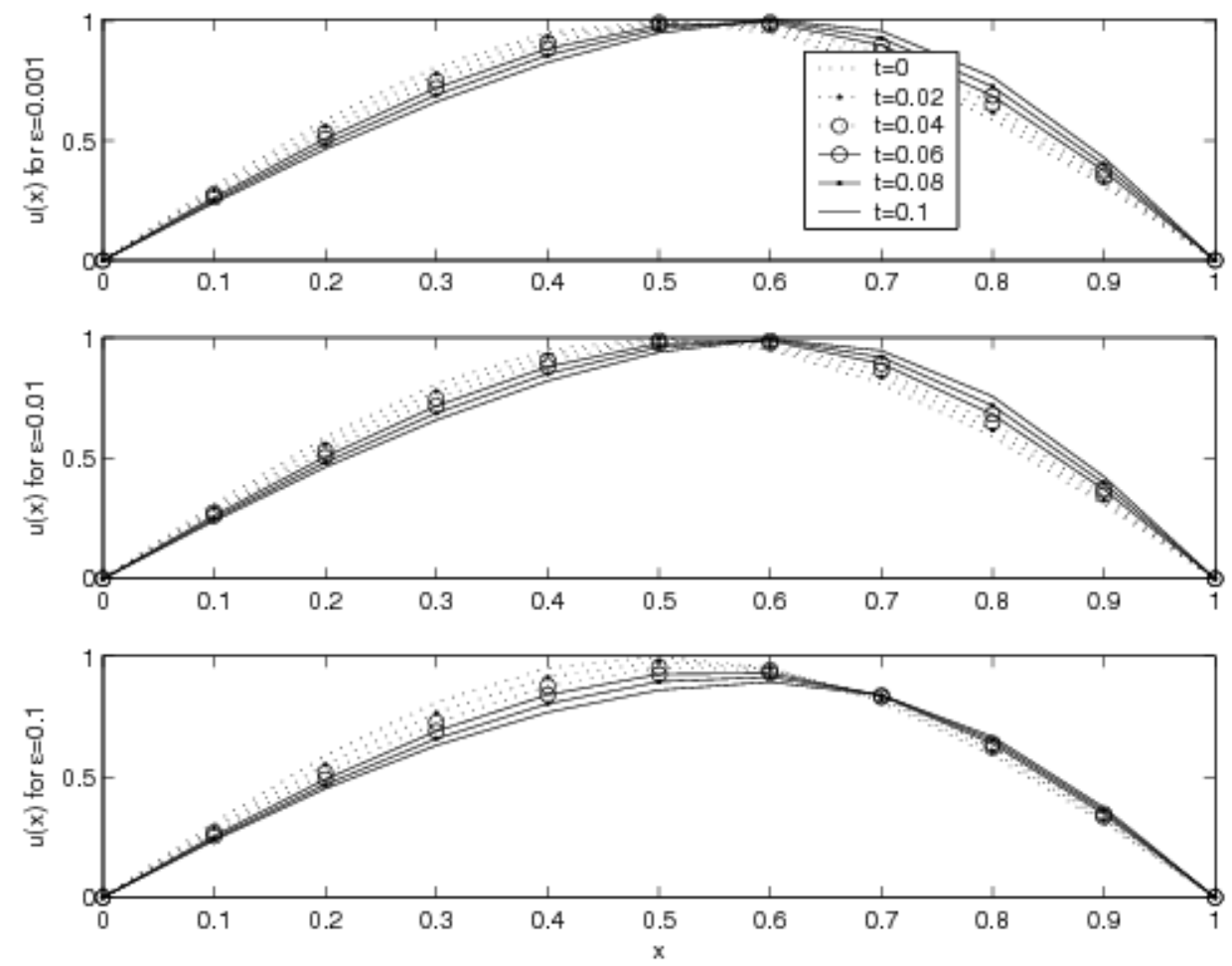

Figure 6. Solution of Burger equation for ( $\beta=0, \alpha=1$, and $\varepsilon=0.001,0.01,0.1$ )

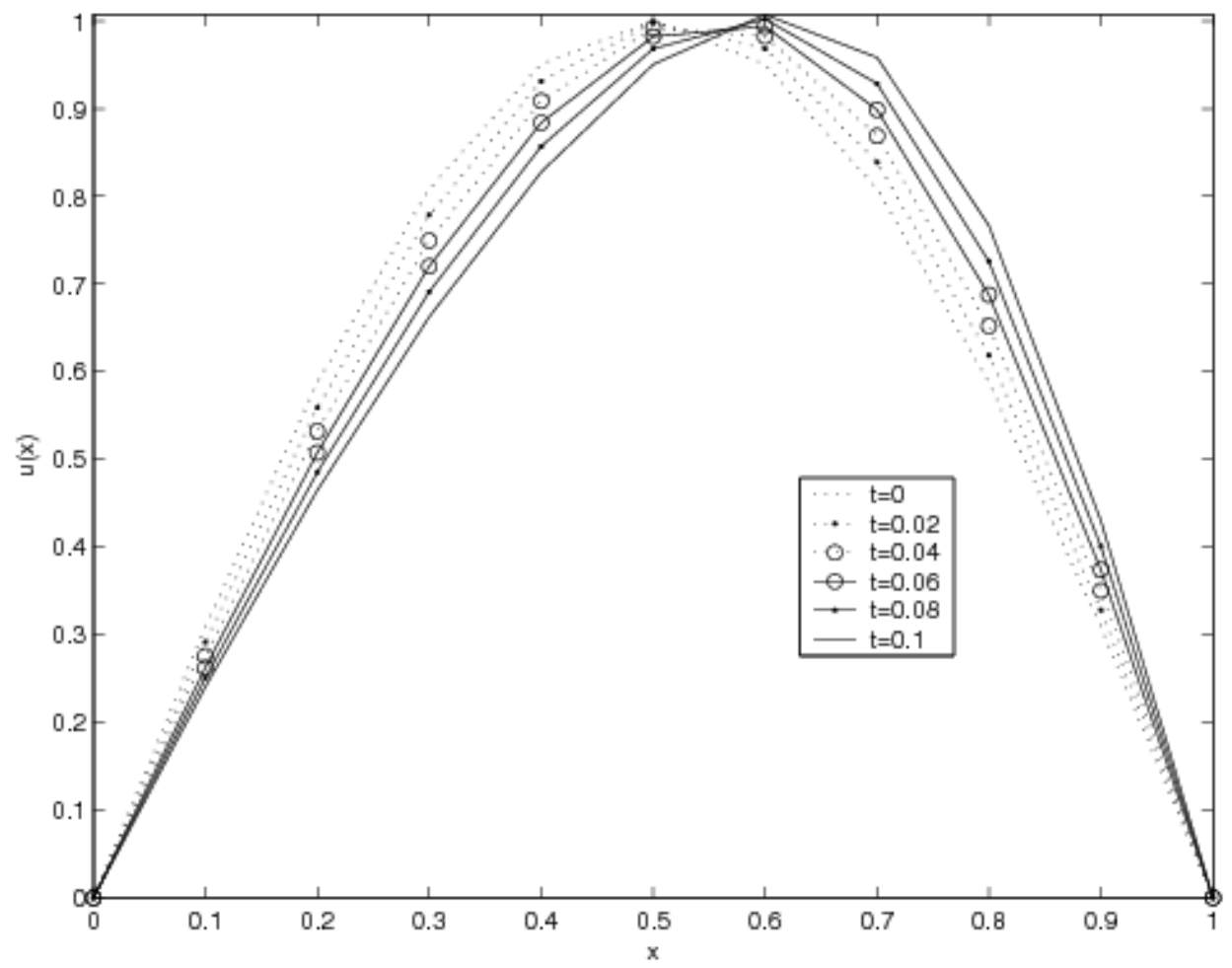

Figure 7. Solution of momentum equation of gas ( $\beta=0, \alpha=1$, and $\varepsilon \rightarrow 0$ )

Model 4: $\alpha=\beta=0$ : The equation (1) resembles heat equation, given by 


$$
u_{t}=\varepsilon u_{x x}
$$

where $u(x, t)$ represents temperature at space $x$ and time $t$. The parameter $\varepsilon$ in this equation defines the thermal diffusivity in the medium. In mathematics, heat equation is the prototypical parabolic partial differential equation. In statistics, the heat equation is connected with the study of Brownian motion via the Fokker-Planck equation. The heat equation is used in probability and describes random walks. For this reason, it is also applied in financial mathematics.

For $\alpha=0, \beta=0$, solution (11) becomes

$$
u_{2}=f_{0}+f_{1} t+\left(.5 \varepsilon f_{1 x x}\right) t^{2}
$$

where

$$
f_{1}=\varepsilon f_{0 x x} .
$$

On solving, this second iterated solution is obtained as

$$
u_{2}=\sin (\pi x)\left[1-\varepsilon \pi^{2} t+\frac{1}{2} \varepsilon^{2} \pi^{4} t^{2}\right] .
$$

The next two successive iterations are

$$
\begin{aligned}
& u_{3}=\sin (\pi x)\left[1-\varepsilon \pi^{2} t+\frac{1}{2} \varepsilon^{2} \pi^{4} t^{2}-\frac{1}{6} \varepsilon^{3} \pi^{6} t^{3}\right], \\
& u_{4}=\sin (\pi x)\left[1-\varepsilon \pi^{2} t+\frac{1}{2} \varepsilon^{2} \pi^{4} t^{2}-\frac{1}{6} \varepsilon^{3} \pi^{6} t^{3}+\frac{1}{24} \varepsilon^{4} \pi^{8} t^{4}\right] .
\end{aligned}
$$

On calculating the higher iterations of the solution, the approximate solutions converges to a closed form $u(x, t)$, given by

$$
u(x, t)=\sin (\pi x) \exp \left(-\varepsilon \pi^{2} t\right)
$$

which is the exact solution of heat equation. The transient solution $u(x, t)$ represented in (21) is a decaying function, i.e., decreasing with the increase of time $t$.

\section{Concluding Remarks}

The nonlinear singularly perturbed time dependent Burger-Huxley equation is solved using VIM, which gives rapid convergent successive approximations of the exact solution. The effect of perturbation parameter in the diffusion term is analysed on the solution. The equation contain nonlinear advection and cubic term. For small values of perturbation parameter, the parameter involved with advection term is more dominant or significant as compared to the parameters involved in the cubic nonlinear term. Therefore, nonlinear advection term plays a significant role in the equation.

The effect of non-linearity parameter $\alpha$ on the solution may not be much significant for smaller values of $\alpha$, say in $(0,1)$. But for larger $\alpha$, beyond 1 , solution may experience a much bigger change towards the far-end of space, particularly in later signals. The changes in the values of two other parameters, i.e. $\beta, \gamma$, may have a little effect on the solution only when $\varepsilon \rightarrow 0$. Batiha et al. $(2007,2008)$ have not discussed about the effect of these parameters.

For particular values of parameters involved the equation reduces to important models for example, FitzHugh-Nagumo equation, Burger equation, momentum gas equation and heat equation. For these four models, the resulting analytical solutions are obtained. The behaviour of solution in space and time is observed numerically for each of the four particular models.

The nonlinear system given by

$$
u_{t}(x, t)-D u_{x x}(x, t)=\beta(1-\alpha u(x, t-\tau)) u(x, t),
$$

is a very significant equation that involves both time delay and diffusion. This equation represents the effect of diffusion and delay on population growth. The approach used in the work presented may be used to solve this system for an analytical solution. This may be a useful topic to explore in the days to come.

\section{References}

Albowitz M. J. , and Clarkson P. A., 1990. Solitons, Nonlinear Evolution Equations and Inverse Scattering Transform, Cambridge University Press, Cambridge.

Al-Khaled K., 2005. Approximate wave solutions for generalized Benjamin-Bona-Mohany-Burgers equations, Applied Mathematics and Computation, Vol. 171, pp. 281-292.

Bateman H., 1915. Some recent researches on the motion of fluids, Monthly Weather Review, Vol. 43, pp. 63-170. 
Batiha B., Noorani M.S.M., Hashim I., 2008. Application of variational iteration method to the generalized Burgers-Huxley equation, Chaos, Solitons and Fractals, Vol. 36, 660-663.

Batiha B., Noorani M.S.M., Hashim I., 2007. Numerical simulation of the generalized Huxley equation by He’s variational iteration method, Applied Mathematics and Computation, Vol. 186, 1322-1325.

Burgers J. M., 1948. A mathematical model illustrating the theory of turbulence, Advances in Applied Mechanics, Academic Press, New York, pp. 171-199.

Cole J. D., 1951. On a quasilinear parabolic equation occurring in aerodynamics, Quarterly in Applied Mathematics, Vol. 9, pp. 255-236.

Duan L. X., and Lu Q. S., 2006. Bursting oscillations near codimension-two bifurcations in the Chay Neuron model, International Journal of Nonlinear Science and Numercal Simulation, Vol. 7, pp. 59-64.

Eckhaus W., 1973. Matched Asymptotic Expansions and Singular Perturbation, North-Holland, Amsterdam.

Eckhaus W., 1979. Asymptotic Analysis of Singular Perturbation, North-Holland, Amsterdam.

El-Wakil S. A., and Abdou M. A., 2008. New applications of variational iteration method using Adomian polynomials, Nonlinear Dynamics, Vol. 52, pp. 41-49.

Evans D. J., and Abdullah A. R., 1984. The Group explicit Method for the Solution of Burgers equation, Computing, Vol. 32 , pp. 239-253.

Feinn D., and Ortolena P., 1977. Catastrophe and propagation in chemical reactions, Journal of Chemistry and Physics, Vol. 67, pp. 2119-2131.

FitzHugh R., 1961. Impulses and physiological states in theoretical models of nerve membrane, Biophysics Journal, Vol. 1, pp. 445-466.

He J. H., 1998a. Approximate analytic solution for seepage flow with fractional derivatives in porous media, Computational Methods in Applied Mechanics and Engineering., Vol. 167, pp. 57-68.

He J. H., 1998b. Approximate solution of nonlinear differential equations with convolution product nonlinearities, Computational Methods in Applied Mechanics and Engineering., Vol. 167, pp. 69-73.

He J. H., 1999. Variational iteration method-A kind of non-linear analytical technique: Some examples, International Journal of Nonlinear Mechanics, Vol. 34, pp. 699-708.

He J. H., 2007. Variational iteration method-some recent results and new interpretations, Journal of Computer and Applied Mathematics, Vol. 207, pp. 3-17.

He J. H., and Wu X. H., 2006. Construction of solitary solution and compacton-like solution by variational iteration method, Chaos Soliton and Fractals, Vol. 29, pp. 108-113.

Hodgkin A. L., Huxley A. F., 1952a. Currents carried by sodium and potassium ions through the membrane of the giant axon of Loligo, Journal of Physiology, Vol. 116, pp. 449-472.

Hodgkin A. L., Huxley A. F., 1952b. The components of membrane conductance in the giant axon of Loligo, Journal of Physiology, Vol. 116, pp. 473-496.

Hopf E., 1950. The Partial differential equation $u_{t}+u u_{x}=u_{x x}$, Communications on Pure and Applied Mathematics, Vol. 3, pp. 201-230.

Inokuti M., Sekine H., and Mura T., 1978. General use of the Lagrange multiplier in nonlinear mathematical physics, in: S. Nemat-Nasser (Ed.), Variational Method in the Mechanics of Solids, Pergamon Press, New York, pp. 156-162.

Ismail, H. N. A., Raslan K., and Rabboh A. A. A., 2004. Adomian decomposition method for Burger's Huxley and Burger's Fisher equations, Applied Mathematics and Computation, Vol. 159, pp. 291-301.

Kadalbajoo M. K., and Patidar K. C., 2002. Spline techniques for solving singularly perturbed nonlinear problems on non uniform grids, Journal of Optimization Theory and Application, Vol. 114, pp. 573-591.

Kaushik A., and Sharma M.D., 2008. A uniformly convergent numerical method on non-uniform mesh for singularly perturbed unsteady Burger-Huxley equation, Applied Mathematics and Computation, Vol. 195, pp. 688-706.

Kaya D., 2003. An explicit and numerical solutions of some fifth-order KdV equation by decomposition method, Applied Mathematics and Computation, Vol. 144, pp. 353-363.

Khattak A. J., 2009. A computational meshless method for the generalized Burger's-Huxley equation, Applied Mathematics Modeling., Vol. 33, pp. 3718-3729.

Liu S. Q., Fan T., and Lu Q. S., 2005. The spike order of the winnerless competition (WLC) model and its application to the inhibition neural system, International Journal of Nonlinear Science and Numercal Simulation, Vol. 6, pp.133-138.

Nagumo J. S., Arimoto S., Yoshizawa S., 1962. An active pulse transmission line simulating nerve axon, Proceedings of the IRE, Vol. 50, pp. 2061-2071.

Nayfeh A. H., 1973. Perturbation Methods, John Wiley, New York.

Ozis T., Aksan E. N., and Ozdes A., 2003. A finite element method approach for solution of Burgers equation, Applied Mathematics and Computation, Vol. 139, pp. 417-428.

Ringhofer C., 1984. On collocation schemes for quasilinear singularly perturbed boundary value problems, SIAM Journal of Numerical Analysis, Vol. 21, pp. 864-882. 
Roos H. G., Stynes M., and Tobiska L., 1996. Numerical methods for Singularly Perturbed Differential Equations, SpringerVerlag, Berlin (1996).

Satsuma J. , 1987. in: M. Ablowitz, B. Fuchssteiner, M. Kruskal (Eds.), Topics in Soliton Theory and Exactly Solvable Nonlinear Equations, World Scientific, Singapore.

Shakeri F., Dehghan M., 2008. Numerial solution of the Klein Gordon equations via He's variational iteration method, Nonlinear Dynamics, Vol. 51, pp. 89-97.

Sleeman B. D., 1982. Small amplitude periodic waves for the FitzHugh-Nagumo equation, Journal of Mathematical Biology, Vol. 14, pp. 309-325.

Stynes M., and O'Riordan E., 1986. A finite element method for singularly perturbed boundary value problem, Numerische Mathematik, Vol. 50, pp. 1-15.

Tatari M., Dehghan M., 2007. On convergence of He's variational iteration method, Journal of Computer and Applied Mathematics, Vol. 207, pp. 121-128.

Wang X. Y., Zhu Z. S., and Lu Y. K., 1990. Solitary waves solutions of the generalized Burger Huxley equations, Journal of Physics A: Mathematical and General, Vol. 23, pp. 271-274.

Zang D. S., Wei G.W., Kouri D. J., and Hoffman D. K., 1997. Burgers equation with high Reynolds number, Physics of Fluids, Vol. 9, pp. 1853-1855.

Zhang G. J., Xu J. X., Yao H., and Wei R.-X., 2006. Mechanism of bifurcation-dependent coherence resonance of an excitable neuron model, International Journal of Nonlinear Science and Numerical Simulation, Vol. 7, 447-450.

Biographical notes

Deepmala Kamboj is an Assistant Professor in Mukand Lal National College, Yamuna Nagar, India. She is teaching graduate mathematics for more than five years. She is also pursuing her PhD from Kurukshetra University.

Dr. M. D. Sharma is a Professor in the Department of Mathematics, Kurukshetra University, India. He has more than 25 years of experience in teaching and research. His current area of research includes Non-linear Differential Equations He has published more than seventy papers in impact factor international journals.

Received May 2012

Accepted March 2013

Final acceptance in revised form March 2013 